\title{
Ciudadanía y autocuidado en salud reproductiva y sexual: Un estudio exploratorio en mujeres trabajadoras de los sectores salud y educación.
}

\author{
Sergio González Rodriguez. ${ }^{1}$ \\ Marcela Arriagada Pincetti ${ }^{2}$
}

\begin{abstract}
Resumen
Se relacionan las temáticas de Ciudadania y Autocuidado en Salud Reproductiva y Sexual refiriéndose un estudio en mujeres que trabajan en las áreas de salud y educación con formación universitaria y técnica. A través de un encuadre metodológico cualitativo basado en entrevistas en profundidad se analizan las prácticas de autocuidado en estas áreas y se constatan las carencias determinadas por creencias, contenidos cognitivo-emocionales y relaciones proveedor/usuaria que inhiben su fortalecimiento. Consecuentemente, se establecen derechos ciudadanos en elámbito de la salud reproductiva, los cuales asegurarian mayores niveles de responsabilidad y de autonomia en el Autocuidado, reforzando el establecimiento de ciudadanias activas.
\end{abstract}

\section{Abstract}

In the article the subjects of Citizenship and Self-caring in terms of Reproductive and Sexual Health relate referring to a study in performed on women working in both the areas of health and education with technical and university levels of formation. Through a qualitative methodological view point based on profoundly performed interviews, self-caring practices within these areas are analyzed and at the same time the deficiencies determined by beliefs, cognitive-emotional contents and provider/user relationships that inhibit it's strengthening are verified. As a consequence, citizenship rights are established in the field of reproductive health, trying to ensure higher levels of resposability and autonomy in Self-caring and reinforcing the establishment of active citizenship.

Palabras Claves:- Autocuidado. -Salud reproductiva y sexual. - Ciudadania

\section{Introducción}

Las Ciudadanías como tema emergente.

El concepto de ciudadania está intimamente relacionado con los procesos de sustantivización de la democracia. Desde esta visión la posibilidad de avanzar en el protagonismo de la sociedad civil se relaciona con los progresos que se alcancen en la extensión de los ámbitos propios de las ciudada- nias, correspondiéndoles participar en la definición de los derechos que le son consustanciales. Las ciudadanias expresan la pluralidad de los diversos actores de la gran trama societal.

En la amplia área de la Salud Reproductiva y Sexual encontramos un conjunto de derechos que deben ser abordados en su institucionalización y legitimación por los y las ciudadanos/as como expresión de la sociedad civil, el Estado y los medios

1. Psicólogo y Antropólogo, Prof. dpto. de Antropología U. de Chile y E. PsicologíaU. de Santiago. sgonzale@abello.dic.uchile.cl

2. Psicóloga, U. de Santiago. 
de comunicación, entre otros. Esta situación se ve afectada por las marcadas restricciones que registra nuestra legislación en contenidos propios de la modernidad inherentes a un desarrollo armónico y coherente. Es así, que los cambios de una creciente modernidad progresan en sus vertientes culturales y en las prácticas de las personas y colectivos sociales mientras existe un retardo en el reconocimiento de estos procesos, los que no alcanzan una respuesta concordante en la «vida institucionalıy en los cuerpos legales que expresan la racionalidad social. De este modo, se observa que se ha producido un sostenido descenso de la tasa de natalidad, un aumento de la escolaridad de la población en general y en particular de las mujeres, una importante adscripción de éstas al mundo laboral y a la vida pública, se han masificado en su uso los medios anticonceptivos modernos y se han instaurado modelos de familias y natalidad deseados. Si bien estos cambios no han llegado con igual intensidad a toda la población, producto de las desigualdades estructurales del país, su prevalencia es indiscutible en las orientaciones de las personas acerca de un deber ser que se legitima social y culturalmente (González, 1998).

En nuestra modernidad la contradicción es evidente en las restrictivas condiciones de debate sobre temas como sexualidad y derechos reproductivos donde se visualizan una serie de prejuicios, estereotipos y opiniones valóricas que no se relacionan necesariamente con lo que piensan, creen y experimentan cotidianamente los diferentes sectores de la sociedad (Solimano,1994). Estas situaciones generan contradicciones evidentes en el plano del ejercicio de la ciudadanía de varones y mujeres. Se constata que en ellas, se dificulta el acceso a información para una conciencia ampliay el posterior ejercicio de los derechos propios en salud reproductiva y sexual. De este modo, se repliegan al espacio de lo privado contenidos que se niegan por una parte en el plano público y, que por otra, desde este mismo ámbito se determinan de manera coercitiva, al prescribirse, por ejemplo, determinados métodos anticonceptivos, excluyéndose otras alternativas y negándose la realidad del aborto in- ducido frente a embarazos no deseados, como situación de hecho.

La salud reproductiva y sexual deben estar consideradas en el plano de los derechos ciudadanos y estar basados en la capacidad de las personas para decidir sobre sus vida sexual y reproductiva. Se hace evidente la necesidad de generar condiciones para asumir una sexualidad sana y la opción reproductiva como una decisión responsable de las parejas ejerciendo un derecho propio.

El debate actual sobre las ciudadanias se encuentra situado en tres lineas de contenidos que son recurrentemente tratadas en sus interacciones reciprocas; la relación individuo-estado, la institucionalidad de las ciudadanías y el reconocimiento en ellas de la diversidad (Favela y Calvillo, 1997). En la realidad latinoamericana las interacciones entre estas tres dimensiones son pertinentes teniendo en cuenta que son discusiones abiertas y con una escasa sintesis concluyente en cada una de ellas. Un ámbito en que se ubica la reflexión que genera el presente trabajo es el del papel del Estado en el reforzamiento, desde lo público, de los medios para ampliar la responsabilidad y la autonomía de las mujeres en la práctica del autocuidado en salud reproductiva y sexual.

El autocuidado en estos ámbitos permite relacionar de manera funcional y de mutuo provecho un proceso de individuación y de resguardo personal que aparece como básico en los contenidos de la modernidad amigable y cercana.

\section{EncuadreTeórico}

\section{El Concepto de Salud Reproductiva}

Las temáticas sobre salud reproductiva son fundamentales para el avance en la calidad de vida de las mujeres y para el ejercicio pleno de sus derechos. Este es un área que se caracteriza por presentar la mayor cantidad e intensidad de problemas de salud que las aquejan. A pesar que esta preocupación es reciente para los organismos de investigación en prevención, actualmente, esta área de la 
salud considera otros aspectos que no la reducen, como tradicionalmente fue, al periodo materno, abriendo una nueva visión respecto a la salud en el plano sexual y reproductivo.

Los conceptos de salud reproductiva sujetos a permanentes modificaciones (por tratarse de un concepto emergente), ayudan a visualizar las variadas dimensiones que implica este tema. Es apreciable la evolución de la primera definición planteada por la Organización Mundial de la Salud (OMS) en el marco de la discusiones sobre los derechos reproductivos. Para este organismo el concepto comenzó a ser considerado de este modo:

"Lasaludreproductivaesunestadodecompleto bienestar fisico, psíquico y social en torno a la reproducción, estoimplicaquelas personastienen lacapacidad de reproducirse y de regular su fertilidad. Las mujeres puedenembarazarse y parirde modo seguro. El resultado delembarazo es exitoso en términos delasupervivencia y bienestardela madre y del niño. Las parejas pueden tenerrelaciones sexuales sintemora embarazos no deseadosodecontraerenfermedades detransmisiónsexual, (OMS,1988)

Podemos apreciar una preocupación por dos dimensiones de la salud en el tema de la reproducción: la prevención, en la elección de métodos de regulación de la fertilidad, y la posibilidad de una vida sexual segura sin temor a infección. Y por otra parte, la intervención, reflejada en la atención dignay adecuada para la mujer embarazada. Estos dos aspectos son trascendentales para la posterior aplicación de politicas de acción en salud con un reconocimiento de autonomía por la persona en el ejercicio de sus atribuciones. Otro aspecto relevante de esta definición es la preocupación por el cuidado de la mujer, aunque limita su consideración al período de embarazo, parto y puerperio.

A pesar que con esta primera definición se produce un avance y se llena un importante vacio respecto al tema también surgen una serie de críticas y cuestionamientos, de diferentes vertientes, en torno a ella. Se plantea que en esta conceptualización no se consideran o, directamente se excluyen, otros aspectos relativos a la sexualidad y la reproducción, especialmente aquellos que formalizan los derechos reproductivos como cualquier otro derecho humano. A partir de estos aportes y de los argumentos esgrimidos al respecto, esta definición es reelaborada en el año 1994 quedando formulada de la siguiente manera:

"Salud reproductiva es un estado de completo bienestar fisico, mental y social, y no solamentela ausencia dedolencias yenfermedades, en todas lascuestionesrelacionadas al sistema reproductivo y a sus funciones y procesos. Lasaludreproductiva, implica entoncesquelas personas están capacitadas parateneruna vida sexual segura y satisfactoria; yquetienen lacapacidadparareproducirse ylatibertadparadecidirsi, cuándo y con qué frecuencia hacerlo. Implicitaen estaúltimacondiciónestá elderechode hombres ymujeres aestarinformados ya teneracceso a métodos de regulación de la fertilidadseguros, efectivos, aceptables y financiables, desuelección. Elderechoalacoesodeservicios desaludapropiados, que permitan alas mujeres unembarazo y parto seguros $y$ les otorguen alas parejas la mejoroportunidad detener un niño o niña saludable»(OMS, en Gysling, 1995).

Esta definición considera la importancia del involucramiento tanto del varón como de la mujer en las decisiones de reproducción y sexualidad, y se amplia elámbito de intervención de los derechos reproductivos, ya que se extiende el campo de decisión a el número, espaciamiento y elección del nacimiento de los hijos. Luego se responsabiliza al Estado en los costos de las acciones y de las politicas dirigidas al logro de estos objetivos en salud reproductiva. En este sentido, su papel es hacer efectivo el acceso para todo ciudadano a la información, al control y al uso de métodos para mantener y/o restablecer, su salud reproductiva.

Posteriormente, en la IV Conferencia Mundial sobre la Mujer realizada en Beijing en 1995, se lleva a cabo la siguiente declaración en torno al tema: "se entiende porsaludreproductiva alacapacidadde disfrutarde una vida sexual satisfactoria y sin riesgo, lacapacidad de reproducirse, y latibertad de hacerlo ono hacerlo, cuándo, y conquéfrecuencia»(ONU,1995).

En este constructo se produce un nuevo avance en la medida que hace referencia a la capacidad de tener acceso a una vida sexual y reproductiva satisfactoria y sin riesgos sin acotación a ningún perio- 
do particular, por lo que esta capacidad es reconocida a lo largo de todo el ciclo vital de las personas, varones o mujeres.

Paralelamente la Organización Panamericana de la Salud introduce en la definición el derecho a tener relaciones sexuales libres de coerción, punto relevante al estar ligado directamente con la elección del individuo, respecto con quien decide relacionarse sexualmente. Este tema debe ser considerado ya que aún es posible encontrar diversas formas de coerción, amparadas en tradiciones socioculturales que limitan al individuo en el ejercicio libre de su sexualidad.

\section{El Tratamientodel conceptode SaludReproductivaen Chile}

En nuestro país la historia del concepto de salud reproductiva y su operacionalización está muy influida por la situación internacional, pero también obedece a factores locales de preocupación en torno a la salud de las mujeres y de sus hijos (Gysling, 1995). Así, temas como la disminución de la morbi-mortalidad materna y el aborto, caracterizaron la preocupación constante en el área de la salud pública, contexto en que nace el concepto de salud reproductiva. Posteriormente, se plantea la necesidad de incorporar a las propias mujeres en una posición activa para influir en la toma de decisiones respecto de su fertilidad.

Es posible constatar que en Chile, el concepto de salud reproductiva también ha sido resultado de una dificil evolución. Al respecto, es posible apreciar que en un inicio la preocupación por el tema de la salud reproductiva estaba centrada en politicas de regulación de la natalidad, pero, hoy en día las perspectivas son mucho más amplias incluyendo la preocupación por la salud sexual de la mujer en sus diferentes etapas de vida.

Actualmente existen diversas instancias (estatales y privadas) en nuestro país, que se preocupan por poner en el debate público los temas de discusión, y velan además por el cumplimiento de los derechos reproductivos de todos los ciudadanos, especialmente de las mujeres (Foro Abierto de Salud y Derechos Reproductivos, 1996).

Estos antecedentes confirman al concepto de salud reproductiva en el ámbito de la ampliación de las ciudadanías. En una primera instancia limitado a la regulación de la natalidad, para posteriormente y con grandes dificultades, hacerlo extensivo a otros temas relacionados con la sexualidad y la reproducción humana.

\section{Dificultades en la Aplicación del Concepto}

En primer lugar, es importante considerar la existencia de un alejamiento entre la conceptualización y la realidad de las diferentes formas de ser mujer (edad, clase, pertenencia étnica, entre otras).

$\mathrm{Al}$ analizar las críticas de las que es objeto el concepto de salud reproductiva, encontramos de dos tipos. Aquellas que tienen relación con las falencias en la conceptualización, y las que se relacionan con la precariedad de los recursos para sustentar los cambios. Entre los factores que acusan las falencias de este concepto encontramos:

a) El binomio Madre-Hijo: Una de las grandes dificultades que ha encontrado la salud reproductiva para convertirse en un práctica cotidiana de las mujeres, la representa la acotación de este término al tema gineco-obstétrico del período fértil. Así, la mujer es considerada objeto de preocupación solamente en relación a su hijo, es decir, en su rol maternal, sin asumirla como agente, con necesidades y problemas propios de su condición de mujer. Además, es valorada sólo en su fase reproductiva, considerándola como reproductoray no como productora, por eso su cuerpo en sí no constituye un foco de interés, salvo en la medida que logre engendrar productos sanos de manera eficiente y sin costos extremados para el sistema (CORSAPS, 1994). Sin embargo, incluso dentro de este binomio, se desatienden las problemáticas que tienen relación directa con la mujer y no así con el 
hijo, esto lo revela la evidente despreocupación de los gobiernos en relación a las muertes, lesiones y discapacidades que sufre la mujer durante y después del embarazo (UNICEF, 1996), y también se manifiesta en la escasez de programas cuyo objetivo sea diferente al período fértil de la mujer. Esta situación demuestra que a pesar de los avances en la conceptualización e intervención en salud reproductiva, estos han sido insuficientes, al no considerar los cuidados de la mujer integralmente a lo largo de toda su vida reproductiva.

\section{b) Excesivo apego al concepto tradicional de familia:}

Los temas de salud reproductiva y planificación de la familia están centrados en una concepción de familia nuclear tradicional, considerada esta última como «unidad fisica, económica y emocionalmente cerrada que goza de estabilidad a largo plazo" (Valdés, 1998). Esta descripción desconoce las notorias transformaciones que ha sufrido la familia actualmente en nuestra sociedad, impidiendo que las familias no normativas sean consideradas en las politicas atingentes al tema de salud reproductiva.

En la actualidad existen diferentes tipos de familia. Están las familias monoparentales (aquellas constituidas por un sólo padre y sus hijos), las familias reconstituidas (aquellas en que se unen en pareja dos adultos con sus respectivos hijos), y otros tipos de familias que se alejan del modelo tradicional nuclear (Molina, 1998). Los modelos emergentes de familia muestran la diversidad respecto a un modelo imaginario, es decir, plantean una serie de cuestionamientos de la realidad asumida como ideal. Al respecto, podemos señalar que varios de los cambios sufridos por la mujer influyen poderosamente en la familia actual. Ellas han pasado a constituir un tercio de la fuerza laboral actualmente existente, lo que modifica el rol que históricamente venian desempeñando. Así, su incorporación a la vida pública ha hecho que sus tareas delámbito privado se compartan con su trabajo remunerado. Esta situación ha producido un reordenamiento de las prácticas reproductivas, mostrando un notable descenso en la tasa de fecundidad, del $5.1 \%$ en 1950 , al 2.6\% al 1995 (CORSAPS, 1994); junto a esto, se modifican las relaciones de poder dentro delámbito familiar en el cual la mujer, antiguamente, ocupaba una posición subordinada y actualmente, con su incorporación al mundo laboral, participa en relaciones más igualitarias (Valdés, 1998). Todos estos cambios marcan cambios estructurales en las familias, las que hoy en día son reconocidas como una alianza opcional y provisoria basada en el afecto (Diaz et al, 1994). En la medida en que se asuma esta nueva realidad se podrán elaborar prácticas más efectivas de intervención en salud reproductiva.

\section{c)Falta de reconocimiento de la importancia} de la vida sexual en las mujeres: Actualmente la salud reproductiva se sitúa en un contexto que separa la sexualidad de la reproducción, situación que influye poderosamente en el reconocimiento del derecho al placer sexual en la mujer (Díaz et.al, 1994). Al respecto, el enfoque actual de salud reproductiva tiene la misión de ampliar su mirada hacia la importancia que juega la vida sexual en la mujer. Esta importancia debe reconocerse y asimilarse socialmente para considerar también los derechos que tiene la mujer en este plano. Además, como ciudadana, cada mujer tiene derecho a ser vista y tratada como la persona única que es, y a expresar su sexualidad con libertad, y a decidir en relación a su conducta reproductiva. Esta perspectiva permite que la mujer asuma como uno de sus tantos derechos el gozar de una vida sexual plenay a la vez se sienta respaldada por :

i) Un sistema de salud que vele por sus intereses no sólo cuando es madre, sino que lo haga a lo largo de toda su vida reproductiva y sexual.

ii) Una sociedad que rompa con las relaciones desiguales de género que le impiden a la mujer tener una actitud activa respecto de su sexualidad (Figueroa, 1995).

En otras palabras debe ampliarse el enfoque excesivamente biologicista centrado en la enfermedad para dar legitimidad a las relaciones sexuales en un contexto de salud, asegurando la igualdad y respeto tanto para hombres como para mujeres, como elementos necesarios para la existencia de 
salud sexualy reproductiva y de los derechos concomitantes.

\section{El ConceptodeAutocuidado}

Este concepto proviene de los ámbitos de la educación y la salud donde es incorporado en torno a la práctica de la enfermería (Garcia, 1994). Aquí se lo conceptualiza y se formula el primer modelo, específicamente en la teoria de autocuidado de Orem (Farfán,1996; Garcia,1994). De este modo se define el autocuidado como "todas aquellas actividades que realizan los individuos, las familias, o comunidades, con el propósito de promover la salud, prevenir la enfermedad, limitarla cuando existe o restablecerla cuando sea necesario" (Orem, en Espinoza y Gazmuri, 1993). La autora plantea que las necesidades de salud son diferentes en cada individuo, por lo cual las clasifica en tres niveles:

a) Necesidades Universales: corresponden a las demandas en salud comunes a todos los seres humanos y que surgen como producto del diario vivir.

b) Necesidades de Desarrollo: corresponden a aquellas demandas que surgen como producto del desarrollo del ser humano a través del ciclo vital, como por ejemplo las necesidades propias de etapas como el puerperio, la lactancia, el embarazo, la adolescencia, la adultez, la senectud, entre otras.

c) Desviaciones de la Salud: son todas aquellas demandas y acciones requeridas por una persona en momentos de enfermedad, agresión y sufrimiento. También se denomina cuidado terapéutico y son acciones perfectamente realizables por la familia, la persona, la enfermera u otro para restablecer el estado de salud en el sujeto.

Al respecto, podemos destacar que existen prácticas de autocuidado aplicables a los diferentes estados y necesidades de salud y que además, puede incorporarse a diferentes actores en la promoción de la salud y las prácticas de autocuidado, tales como, la familia, amigos u otros agentes. En caso de que la persona no pueda cuidarse por si misma, se organiza la atención de los sistemas de cuidado de la salud los que introducen acciones voluntariamente seleccionadas por los individuos para satisfacer las necesidades de autocuidado propias o de los demás, incluyendo sistemas de autocuidado y cuidado dependiente, creados para satisfacer necesidades de salud en el contexto de la vida diaria, como también en casos de desviaciones de salud. En otras palabras, uno de los principales beneficios del método de autocuidado es revertir la dependencia que obliga al usuario a consultar a los sistemas de salud. En este sentido podemos destacar que esta perspectiva rompe con los modelos tradicionales de atención en salud, donde el beneficiario dependia completamente de la acción y consejos del proveedor de salud (OPS, 1992b). El autocuidado permite, por tanto, fortalecer los niveles de autonomia y de auto-responsabilidad ante si mismo y ante otros, lo cual permite pasar de la lógica de sujeto/objeto de atención de salud a ciudadanos que están conscientes de sus necesidades con estrategias propias para velar por su salud y la de la comunidad. Se rompe el inmovilismo de tomar a la persona como un receptor de acciones de salud. En este sentido el autocuidado como método de preocupación activa por el bienestar es rápidamente extrapolable para reforzar la actitud activa en el campo de los derechos y autodeberes de las personas.

La incorporación del modelo de autocuidado permite además, ampliar la cobertura de acción en todos los planos de la salud, sin embargo, tradicionalmente en salud y más especificamente, en medicina, la dinámica de intervención no ha dado espacio para la acción del sujeto, por lo cual, este modelo ha encontrado varias dificultades para manifestarse en la práctica, ya que históricamente, se ha dejado de lado la subjetividad de los individuos sin asumirla como un factor influyente en los modelos de intervención en salud (Espinoza y Gazmuril, 1993). Actualmente, y con la incorporación del método de autocuidado en salud, se ha facilitado a las personas tener un grado de participación mayor que les permite ser actores de su proceso de cuidado y salud personal. Para el logro de 
este objetivo, el proveedor de salud debe reconocer e identificar las prácticas de autocuidado que ya tiene cada sujeto, para luego enseñarle nuevas estrategias que se complementen con las que ya posee. Esta técnica ha resultado ser una herramienta eficaz para el cuidado y tratamiento de diversas enfermedades tanto de carácter terminal como de orden crónico. No obstante, este modelo de intervención puede ampliar su rango de acción a los niveles preventivos en problemas de salud social o psicológica. Esta situación plantea un desafio abierto a asumirse en el próximo siglo (Tapia etal, 1996) cuyo objetivo es ampliar las áreas de intervención del autocuidado hacia los ámbitos psicológicos y sociales que influyen, también, en la salud de los individuos. Al respecto la OPS (1992a) plantea el autocuidado como un frente de toma de decisiones y de autodeterminación a nivel individual que contribuye a desarrollar prácticas específicas a nivel social, consecuentes con la teoría de género. El autocuidado se entiende, desde este enfoque, en la doble dimensión de lo individual y lo colectivo como polos indisociables que se retroalimentan a través de diversas expresiones. En lo individual permite la reafirmación del yo; sin embargo, esta dimensión no es reductible a su fisiologia, lo individual implica una concepción holística de la especie humana. En lo colectivo, propende al reconocimiento del saber y la práctica que las personas tienen en relación a las acciones que favorecen la salud. Esta visión pone en relieve los componentes individuales, tales como, el proceso de toma de decisiones y la autodeterminación, que deben estar presentes en el autocuidado. A su vez, introduce la importancia de los componentes sociales como la provisión de recursos que sustenten las decisiones de autocuidado.

Respecto a las condiciones que deben darse para que se ejecuten las prácticas de autocuidado en la vida diaria se plantea que lo fundamental es hablar de "actores genéricos» para iniciar actividades a nivel de politicas y programas en salud en el plano del autocuidado. Esa visión más ampliada permite concentrar los esfuerzos de autocuidado en los planos sociales e individuales. Bajo este punto de vista es destacable la relación de similitud entre el constructivismo y el autocuidado, fundamentalmente, en un marco referencial de las formas de promover la salud, hasta el momento practicada por la medicina. Es importante destacar el rol de constructor del conocimiento que este modelo le asigna al agente (Sepúlveda, 1991). Este planteamiento destaca los contenidos de la salud como parte de un estilo de vida.

\section{Metodologia}

\section{Antecedentesy Muestra:}

En el presente estudio se evalúan las prácticas y comportamientos de autocuidado en un grupo de mujeres que supuestamente deberían tener un mayor y mejor involucramiento en procesos de autocuidado en salud reproductiva y sexual. Desde este supuesto se conformó la muestra optándose por mujeres que se desempeñan profesional y laboralmente en las áreas de salud y educación, partiendo de la base que ellas tendrian por su formación universitaria o técnica, y por su desempeño laboral/profesional una mayor accesibilidad a conocimiento sistemático e información sobre estos ámbitos, y por lo tanto una mejor práctica.

La investigación es de carácter exploratorio, a nivel transversal, al no existir estudios anteriores en estos segmentos de trabajadoras con similar preocupación temática. Además se consideró que la reciente aparición de un concepto integral y multidimensional de salud y derechos sexuales y reproductivos podría influir en la falta de información y de práctica en elárea.

La muestra estuvo conformada por 16 mujeres de 20 a 60 años de edad, quines llevan trabajando un mínimo de dos años en servicios de salud y educación. Se conformaron cuatro grupos muestrales: dos de mujeres del sector salud y dos de trabajadoras del sector educación. En ambos sectores se distinguió un grupo con formación universitaria y uno con formación técnica. 
El principal instrumento de investigación consistió en una entrevista en profundidad semiestructurada. En un primer momento, se definieron las unidades de significación relevantes que dieron origen a la pauta de entrevista, la cual fue testeada en mujeres no consideradas posteriormente como parte de la muestra.

Unidades de significación establecidas:

Se determinaron las siguientes unidades de significado:

- Medidas y Prácticas de Autocuidado: Acciones que generan, mantienen, intensifican o restablecen el autocuidado.

- Conocimientos (manejo de información) sobre salud sexualy reproductiva: Corresponde a los conocimientos o información respecto a las dimensiones que abarca la salud sexualy reproductiva. Esta variable incluye temas como: conocimientos en relación a métodos anticonceptivos (propiedades-eficacia-efectos secundarios), enfermedades de transmisión sexual (ETS) y virus de inmuno deficiencia adquirida (VIH/SIDA), manejo de información respecto al uso del preservativo, cuidados que requiere un embarazo (controles), terapia hormonal de reemplazo, y ciclo menstrual.

- Obstaculizadores del autocuidado: son aquellos componentes que impiden o dificultan elautocuidado de la salud reproductiva en las mujeres.

. Facilitadores del autocuidado: son aquellos elementos que facilitan o promueven el autocuidado de la salud reproductiva en las mujeres.

\section{Análisis de resultados yconclusiones.}

Se presentan de manera integrada los resultados y las conclusiones del estudio estableciendo una linea de continuidad entre ambos. Se previlegia, de este modo, la relación e interpretación de los discursos de las entrevistadas. A continuación, se refieren los contenidos agrupados en las áreas temáticas más relevantes que aporta la investigación:

\section{Las mujeres consultadas realizan prácti- cas de autocuidado»:}

\section{a) Tipos deprácticas deautocuidado}

Se confirma la existencia de prácticas de autocuidado de las mujeres consultadas. Entre las prácticas de autocuidado en salud reproductiva más frecuentes encontramos:

Asistencia a controles ginecológicos: realización de exámenes preventivos (papanicolaoumamografias), utilización de métodos anticonceptivos, planificación familiar, autopalpación mamaria, registro de periodos menstruales, mantención de la higiene. Sin embargo, se observa insuficiencia en la calidad y periodicidad de las prácticas, ya que no siempre son realizadas con la regularidad y sistematicidad que requieren. Las prácticas de autocuidado en el área reproductiva requieren de periodicidad, es decir, deben realizarse periódicamente según las instrucciones del especialista.

En relación a la calidad de las prácticas nos referimos a que las mujeres no necesariamente llevan a cabo las prácticas de autocuidado con la rigurosidad que requieren, por ejemplo, asisten a sus controles médicos, pero no consultan sus dudas sobre los procedimientos que deben seguir, o utilizan un método anticonceptivo, pero no lo controlan. Al respecto, los antecedentes que motivaron la investigación son relevantes porque encontramos que mujeres con un mayor acceso a formación e información presentan «inhibiciones y olvidos» importantes en sus prácticas de autocuidado. Las razones se reiteran sobre prejuicios y temores determinados culturalmente, como a su vez, la falta de preocupación sistemática individual. Esto es grave teniendo en cuenta que las prácticas de autocuidado no son suficientemente efectivas si no se llevan a cabo con regularidad.

b) Inexistencia de prácticas de Autocuidado entre grupos:

No se presentan diferencias en las prácticas de autocuidado en las mujeres según su formación y tampoco se identifican diferencias de acuerdo a los 
sectores laborales a los que pertenecen. Esto permite llegar a la conclusión que las mujeres adquieren conocimiento en torno a su salud sexual y reproductiva de otras fuentes, ya sean formales como informales.

Podemos señalar que existen elementos comunes de acuerdo al sector al que pertenece la mujer, que obstaculizan las prácticas de autocuidado que ellas llevan a cabo. Las mujeres del sector salud describen factores como los turnos, la carga laboral y el estrés psicológico que les genera su trabajo, los que impiden que realicen sus prácticas en salud reproductiva con la regularidad que requieren. Del mismo modo las mujeres del sector educación describen elementos como el cumplimiento de metas, la excesiva cantidad de horas de trabajo y la tensión, como algunos de los factores que entorpecen o dificultan su autocuidado. Se aprecia una gran coincidencia en los factores expuestos como obstaculizadores del autocuidado, expresados por las mujeres con y sin formación universitaria de ambos sectores. Esto nos indica que en el caso de esta muestra, los elementos señalados por ellas están impidiendo sus prácticas de autocuidado en salud sexual y reproductiva, independientemente del sector donde se desempeñan laboralmente y de la formación profesional que hayan recibido.

c) Las diferentes necesidades de autocuidado:

Existe concordancia entre los resultados del instrumento cualitativo y la teoria sobre diferencias en las necesidades de autocuidado. Las mujeres asocian el autocuidado de la salud reproductiva a diferentes tipos de necesidades, es así, que encontramos en la noción de autocuidado una asociación del término con distintos contenidos que surgen durante las etapas de vida (necesidades de desarro1lo) como los cuidados durante el embarazo, menopausia. Las necesidades diarias de autocuidado (necesidades universales), como la higiene, y por otra parte, las necesidades de autocuidado cuando peligra la salud (necesidades de déficit), como la asistencia a los controles médicos por urgencias ginecológicas. Esto confirma que las prácticas de autocuidado varían según el tipo de necesidades a las que responden.

\section{2. ¿Existen variables de género que influyen en las prácticas deautocuidado de las mujeres de ambos grupos»}

\section{a) Caractenísticas de personalidad:}

Aquellas que son adscritas a la mujer mediante la socialización y el perfil cultural dominante. Al respecto, podemos señalar que las mujeres hacen alusión reiteradamente a la postergación, la dejación, la pasividad y la vergüenza como características que explican la ausencia de conductas y prácticas de autocuidado en sus coetáneas. Las mujeres perciben la influencia de estas características en la incapacidad de símismas y de otras mujeres para realizar prácticas de autocuidado de la salud reproductiva.

La postergación y la dejación en la mujer se explican de acuerdo a la socialización en la labor de servicio a otros, la que influye en que priorice las necesidades de su entorno por sobre las propias. La pasividad y la vergüenza inhiben la autovaloración de los propios procesos de salud o de perdida de ésta, incluso en estas mujeres, que por formación podrian expresar una mayor asertividad en temas que cognitivamente les son cercanos. Las variables emocionales y afectivas determinan los compromisos de los comportamientos de autocuidado.

b) Relación Proveedor/a - usuaria:

Podemos destacar además la influencia de variables de género en la relación que establece la mujer con el/la proveedor/a de salud, donde la mujer asume un rol notoriamente pasivo, en términos de obedecer las órdenes que se le administran, sin consultar cuando tiene dudas y paradojalmente sin consultar por temor a ser catalogada de ignorante. Esta relación paradojal refleja que la mujer sólo informa lo que asume como "de interés para el proveedor/a de salud", ocultando o restringiendo información que puede ser importante para el diagnóstico o la intervención. Estas mujeres «califica- 
das técnicamente» refieren dificultades e incomodidades psicológicas para asumir una actitud activa en el marco de la atención en salud reproductiva y sexual cuando son pacientes o usuarias de los servicios de salud.

Al diseñar nuevas estrategias en los programas de autocuidado es necesario incorporar la dimensión de ejercicio ciudadano. Esta se refuerza al tener las/los usuarias/os los resguardos que les permitan mantener una actitud activa en la lógica del ejercicio de derechos inherentes y apropiados para que los servicios de salud cumplan su objetivo.

\section{c) Autovaloración positivay Autocuidado:}

Entre los factores que facilitan el autocuidado podemos destacar que una autovaloración positiva es una de las características más señaladas por las entrevistadas como motivador de su cuidado personal, no sólo en elárea de la salud reproductiva, sino que en la salud en general. Sin embargo, es destacable la reproducción de una impronta cultural donde la autoestima positiva está asociada en las mujeres a la prolongación de la labor hacia otros cercanos, donde el cuidado de sí misma pasa a ser un medio para mantener el cuidado y protección de otros. En otras palabras la mujer relaciona y justifica su cuidado personal en cuanto cuidadora de otros.

\section{d) Validación del Autocuidado durante la} etapafértil:

Existe también un elemento que es tanto facilitador como obstaculizador del autocuidado, nos referimos a la creencia de que el autocuidado es válido en ciertas etapas de vida de la mujer, especialmente durante la maternidad. Si bien esta creencia acerca a muchas mujeres a los servicios de salud para mantener su cuidado personal durante la fase reproductiva, influye en su alejamiento durante la etapa post-reproductiva, ya que se cree, que con la disminución de su vida sexual y la inexistencia de fecundidad, ya no requieren de cuidados particulares en su salud reproductiva. En relación a este punto debemos destacar que uno de los obstáculos de la salud reproductiva es la vinculación del autocuidado de la mujer al binomio madre-hijo. En este sentido, es destacable que las mujeres señalan la importancia de los cuidados de la salud reproductiva ligados a esta etapa, no obstante, reconocen que en los otros períodos del ciclo vital la preocupación decae significativamente.

Entre los derechos de las mujeres que deben explicitarse en relación con la salud reproductiva y sexual está la necesidad de catalizar un cambio cultural inducido en pos de entender de manera integrada la sexualidad a la vida normal de las personas. Las politicas públicas deben posibilitar la extensión de los principios de la modernidad donde el Autocuidado no puede quedar cercenado por la creencia que la sexualidad sólo es efectiva en la etapa de fertilidad y válida exclusivamente cuando es conducente a la reproducción.

\section{Existen relacionesambivalentesentrelos} factores facilitadores y obstaculizadores del autocuidado»

\section{a) Actividad Laboral y Autocuidado:}

La actividad laboral traducida en la jornada, el cargo, las tareas, y el exceso de trabajo, son factores que generan cansancio, interviniendo en la disposición con que las mujeres asumen sus prácticas de autocuidado. Conjuntamente, y a pesar de los aspectos negativos, destacan una positiva influencia en las prácticas de autocuidado, los temas y casos que tratan en su actividad laboral, los que están altamente relacionados con la salud reproductiva, pudiendo acceder a información veraz y oportuna que las acerca a una toma de conciencia mayor respecto a las situaciones de riesgo. En otras palabras, las trabajadoras de educación y salud reconocen en su actividad laboral una instancia de formación y una fuente de conocimientos sobre salud sexual y reproductiva.

\section{b) Interferencia defactores contextuales de losservicios de salud:}

La interferencia producida por la influencia de factores contextuales fisicos y psicológicos de los servicios de salud, como la deficiente calidad de la atención, falta de recursos humanos y materiales, 
duración prolongada en los tiempos de espera, son aspectos que inciden en el alejamiento de las mujeres de los servicios de salud, y por ende, en la postergación de su autocuidado. Unido a esto encontramos factores como la acción castigadora de algunos/as proveedores/as de salud y la mala calidad de la comunicación proveedor/a-usuaria, que también obstaculizan las prácticas de autocuidado. De igual modo, y de manera positiva estos factores son señalados como potenciales facilitadores del autocuidado ya que si estuvieran presentes en condiciones óptimas facilitarian y estimularian la realización de prácticas de autocuidado.

De acuerdo a la OPS (1992a), existe una relación indisociable entre los factores individuales, como la toma de conciencia en relación al riesgo en salud reproductiva, y la preocupación pública de organismos sociales que sustentan los recursos para que el sujeto tenga la posibilidad de materializar sus decisiones. Es así como el mejoramiento de los sistemas de salud es fundamental, para que en el plano individual, se refuerce la opción de velar por la propia salud, de otra forma la promoción del autocuidado como estrategia se ve debilitada. Nuevamente es apreciable la importancia de la acción positiva del Estado en la promoción de la responsabilidad consigo mismo que implica el autocuidado. Estos contenidos dialógicos que impactan directamente la autonomía y la responsabilidad individual permitirian desde este plano constituir ciudadanías en que se encuentre, lo privado (lo propio, lo intimo) y lo público (lo social, lo politico).

\section{Existen relaciones paradojales entrelas creencias y las acciones deautocuidado":}

Al respecto podemos señalar que las relaciones paradojales revelan una contradicción entre dos factores mutuamente excluyentes, y cuya interacción es contradictoria ya que obstaculiza o facilita el autocuidado.

\section{a) Omnipotencia frente al riesgo:}

La frecuente actitud de «a mí no me va a pasar", es reiterativa en las explicaciones dadas por las entrevistadas respecto a su despreocupación por el autocuidado en salud reproductiva. Ellas, reconocen en síy en otras mujeres la creencia pre-racional (debido a que no argumentan explicaciones congruentes y lógicas) de estar alejadas del riesgo. Esta situación se complejiza en el tema sexual, específicamente en torno a las relaciones sexuales y el posible contagio de ETS y VIH, debido a que la relación sexual conlleva elementos afectivos y fisiológicos que requieren de un contexto de entrega donde no hay cabida para el riesgo. En este sentido, está presente el «mito del amor romántico». Se produce también, la negación del riesgo, donde no es necesario prevenir porque de acuerdo al sujeto (pareja), el riesgo no existe.

\section{b) Temor al diagnóstico negativo, y portan- to, inasistencia a controles y exámenes preventi- vos:}

Se produce una relación paradojal entre el temor a ser diagnosticada con alguna enfermedad terminal (cáncer de útero o de mama) y la realización de exámenes preventivos. De acuerdo a las entrevistadas, el temor a ser diagnosticadas con una enfermedad grave genera miedos que son muy dificiles de vencer obstaculizando la ejecución de exámenes preventivos.

\section{c) Pareja estable como método de preven- ción de contagio de VIH/ SIDA:}

Otra de las situaciones contradictorias que surgen en torno al cuidado de la salud reproductiva, se relaciona con la creencia generalizada que mantener una pareja sexual estable, es un método infalible para la prevención de VIH/SIDA. Al respecto, se evidencia una confusión sobre la información entregada por las campañas preventivas debido a que si bien la mantención de una pareja estable es una forma de prevenir el contagio con VIH, existe excepciones donde ésta forma de prevención no es una manera infalible de evitar el contagio, en otras palabras, no es un método preventivo autónomo, sino que debe ser acompañado de otras medidas. Resulta alarmante evidenciar que estas mujeres, que suponemos que son las que expresan el mayor ni- 
vel de formación e información en los temas de salud reproductiva y sexual, confirman con absoluta seguridad que la mantención de una pareja estable es una de sus prácticas de autocuidado relacionadas con la prevención de $\mathrm{VIH}$. La relación resulta paradojal, debido a que si bien esta medida es una forma de prevención del contagio de esta enfermedad, no asegura su eficacia por no depender directamente de ellas.

En relación a este punto es cuestionable tanto el rol formador y la información entregada por los medios de comunicación como las campañas públicas provenientes de organismos estatales. Estas deben asumir un compromiso educativo respecto a los contenidos que imparten al público en general. En este sentido, al elaborar los programas de prevención e intervención deben tomarse en cuenta los déficits de formación y la pluralidad del público receptor, tratándose a los ciudadanos en su calidad de tales, y no como incapacitados para acceder a la totalidad de la información cuando se expresa de manera consistente y eficaz.

\section{d) Incapacidad de hablar abiertamente de sexualidad:}

Este es uno de los factores que obstaculiza en gran medida las prácticas de autocuidado, donde tomar conciencia y evitar conductas de riesgo, asumir decisiones informadas, entre otras, se ven limitadas por la falta de información disponible sobre sexualidad, derivándose todo esto de la incapacidad de reconocer el tema, social e individualmente, asi como el temor a la sanción social. Al respecto es constatable la existencia de un doble discurso social, donde a pesar de la evidencia que indican la necesidad urgente de reconocer la sexualidad adolescente, la existencia de familias no tradicionales y los fenómenos que inciden en la morbi-mortalidad por ETS y VIH, existe una invisibilidad de la presencia y repercusiones de estos fenómenos.

\section{e) Especificidad del lenguajemédico:}

El discurso de las mujeres revela que perciben un distanciamiento entre su realidad (significados y conocimientos) y el lenguaje médico. La asocia- ción del concepto de salud reproductiva con imágenes médicas y lenguaje especializado, impide una comprensión acabada de los procedimientos e intervenciones médicas y del significado del autocuidado en la salud sexual y reproductiva. La tecnificación del lenguaje afecta en la comprensión de las mujeres obstaculizando la actitud activa respecto a su salud. No olvidemos que estamos ante grupos muestrales con calificación en esta temática, y de igual modo, se presentan estas situaciones que se podrian suponer, exclusivamente, en otros tipo de mujeres sin formación especifica.

Como hemos apuntado con anterioridad este hecho se relaciona con el ejercicio de ciudadanías pasivas en que se reproduce la concepción del ciudadano como receptor de políticas públicas y de "servicios" que no sólo no comprende y controla, sino que sus significados e impactos le son distantesy ajenos.

\section{5 «xiste una desexualización dela salud sexual y reproductiva»}

\section{a) Salud sexual y reproductiva como un área compleja de la salud:}

La salud sexual y reproductiva no puede ser concebida de forma semejante a como se consideran otras áreas de la salud, específicamente en salud sexual encontramos una infinidad de elementos sociales y culturales, que dan vida a los conocimientos y actitudes que manejan las personas sobre salud reproductiva y salud sexual. Al respecto y ligado a la incapacidad de asumir social e individualmente la sexualidad encontramos una desexualización de la salud sexual y reproductiva, donde las prácticas de autocuidado señaladas por las entrevistadas se relacionan exclusivamente con métodos de cuidado de la salud reproductiva, dejando de lado los métodos y acciones que preservan la salud sexual. Es así como conductas tales como el uso de preservativo para evitar contagio ETS-VIH/SIDA, la realización del test de E.L.I.S.A.(detección de VIH/SIDA), la elección de la pareja sexual, la relación sexual libre de coerción, la gratificación en la vida sexual, son elementos omitidos o no considerados como parte de la salud 
sexual, por las mujeres entrevistadas.

Esto es revelador por la naturaleza de la muestra y la intención del supuesto inicial de que damos cuenta. Si mujeres con instrucción, universitaria o técnicay que pertenecen a los mundos laborales de la salud y la educación presentan un bajo nivel de formación e información en salud sexual y reproductiva, otras mujeres que no cuentan con esta cualificación se encontrarán en situación desmejorada comparativamente, por lo cual, las prácticas de autocuidado en estos ámbitos se encuentran en situación desfavorecida. Emprender acciones urgentes por remediar este estado de cosas es absolutamente pertinente.

\section{b) Faltadereconocimiento delavidasexual en lamujer:}

En relación a este punto podemos destacar la falta de reconocimiento de la vida sexual en las mujeres, existiendo una diferenciación entre el rol de la reproducción y la sexualidad. Las mujeres consultadas conciben la salud sexualy reproductiva como dominios diferenciados cognitivamente. La omisión de factores de salud sexual como los anteriormente señalados responden a esta negación de la sexualidad, lo cual es asumido por las mujeres cono naturaly apropiado para su sexo. Se interpreta este estado de desexualización donde las mujeres no reconocen la necesidad de una situación de bienestar en su sexualidad, en consistencia con no reconocer la carencia de salud en este nivel, carencia que puede ser desplazada a otras dimensiones.

\section{Existeuna evasión de las conductas pre- ventivas»}

\section{a) Recursosnecesarios paralas prácticas de autocuidado:}

Las mujeres hacen referencia a factores económicos y a la disponibilidad de tiempo como recursos básicos que son necesarios para llevar a cabo las prácticas de autocuidado. A ello complementan el manejo de conocimientos pertinentes para realizar exámenes preventivos.

\section{b) La incomodidad de los procedimientos ginecológicos:}

Existe consenso en que la incomodidad de los procedimientos ginecológicos es un factor inhibidor para realizar de manera regular estos exámenes preventivos. Los procedimientos se relacionan con el manifiesto malestar que producen en las mujeres, por la intromisión en la privacidad y el sentimiento de exposición que generan. Esta situación, semejante al caso de los varones, se constituye en uno de los principales obstaculizadores del autocuidado de la salud reproductiva que relatan las consultadas, especialmente en el caso de la visita al médico.

\section{c) La negación del riesgo:}

Existe un temor permanente a enfrentar enfermedades terminales o crónicas del aparato reproductor (como cáncer o VIH/SIDA), lo cual inhibe las acciones de prevención. Esta situación resulta paradójica debido a las consecuencias que conlleva como fenómeno de evasión del riesgo. El factor emocional no permite actuar racionalmente, esto es frecuentemente destacado por las entrevistadas. El temor relativo al contagio de VIH/SIDA confirma esta situación, ya que, las mujeres manifiestan mayores temores al contagio de VIH, el cual tiene una menor prevalencia que el cáncer de cuello o de mama, donde, sin restarle importancia, y a pesar de la asociación de la mujer con el rol reproductor, las connotaciones del contagio de VIH son mucho mayores, en cuanto al impacto social que genera.

\section{d) Experiencias devida:}

Existe una diferencia marcada entre las mujeres que no y las que si han tenido experiencias de vida traumáticas en elárea de la salud, tales como accidentes graves, antecedentes familiares de cáncer, presencia de enfermedades terminales o crónicas, lo cual modifica, en alguna medida, la valoración de la propia salud. Esta situación no necesariamente está ligada a la calidad y cantidad de prác- 
ticas de autocuidado que las mujeres realizan, pero sí marca una diferencia en la forma de valorar la salud.

\section{Dos Propuestas:}

\section{a) Facilitar el acceso de la mujer a los servicios de salud:}

El reconocimiento de factores que influyen en las conductas de autocuidado de las mujeres es un elemento que debe tomarse en consideración a la hora de elaborar proyectos de intervención en salud que pueden acomodarse a la mujery no ser ésta quien se acomode a ellos. Proponemos mejorar la ofertas del sistema de salud en cuestiones concretas como reelaborar politicas de atención, en este sentido, es clara la necesidad de aumentar la calidad de los servicios de salud, especialmente en lo que se relaciona con la pérdida de tiempo que le significa a las mujeres, y en la calidad de la interacción del proveedor de salud respecto a sus usuarias. Se debe optimizar el uso del tiempo que dedican a si mismas, por lo cual los servicios deben facilitar esta tarea, de igual modo, se puede utilizar el tiempo que se requiere para esperar la atención aprovechándolo para informar a la mujer de los riesgos posibles y de las formas en que ella puede acceder a cuidar su salud reproductiva.

\section{b) Iniciar programas de educación en salud sexual y reproductiva:}

Otro aspecto a considerar es la necesidad de fomentar la educación sobre sexualidad y salud sexual y reproductiva. En este sentido revelamos la urgente necesidad de contrastar los mitos en torno al sexo con información oportuna y veraz. De aquí surge un desafio para los sistemas de salud y educación ya que ambos realizan una tarea formadora que puede ser de gran ayuda a la hora de entregar formación e información a las personas. Esto se lograría mediante la capacitación del personal de salud (a todo nivel) respecto a técnicas de autocuidado de la salud reproductiva con niveles de información e intervención diferentes en jóvenes y adultos.

La labor de los sistemas educativos y de salud debe concentrarse en el plano de la prevención invirtiendo recursos en esta área para masificar la promoción del autocuidado como una técnica de salud que promueve la autogestión del estado de salud. Así, el Estado estará promoviendo en mujeres y varones, además, a través del Autocuidado, los valores de autonomía y de individuación que se constituyen en base para la ampliación de las ciudadanías.

\section{ReferenciasBibliográficas}

Agüero M.(1991) «La historia de una niña que al parecer adquirió el concepto de autocuidado». EPAS, Vol VIII, N×3, 23-26.

Arriagada, M. (1999) "Autocuidados en Salud Sexual y Reproductiva: Un Estudio Exploratorio con Trabajadoras de los Sectores Salud y Educación». Santiago. Escuela de Psicología. Universidad de Santiago. Tesis de Titulación en Psicología.

CORSAPS. (1994) «Sexualidad, reproducción y servicios de salud: hacia la construcción de derechos». Santiago.

Díaz S; Sánchez S.; Silvamonge M. (1994) «Realidades y desafios, reflexiones de mujeres que trabajan en salud reproductiva». Santiago. ICMER.

Espinoza M. y Gazmuri P. (1993) «¿Cómo son las prácticas de autocuidado de las madres de niños menores de cinco años controladas en el consultorio Los Castaños frente a la morbilidad diarreica infantil?. Santiago: Universidad Católica de Chile. Tesis para optar al grado de licenciado en enfermería.

Favela, A. y Calvillo (1997) «El Ciudadano en el Mundo Posmoderno". En Revista: Estudios Politicos. Nº 15. pp.53-69. México.

Farfán M. (1996) «Conocimientos y medidas de autocuidado en el climateriom. Santiago: Universidad Católica de Chile. Tesis para optar al grado de licenciado en enfermería.

Figueroa G. (1995) «Aproximación al estudio de los derechos reproductivos". Programa de salud reproductiva y sociedad. México. Año 1, N×8, 5-28.

Foro Abierto deSalud y Derechos Reproductivos (1996) «Conferencia Internacional de Población y Desarrollo, El Cairo 1994». Material del Foro de Salud y Derechos reproductivos y la Red Nacional de Investigación Acción.

García M. (1994) «Aplicación del modelo de autocuidado en la atención de enfermería del servicio de urologia del Hospital Cli- 
nico de la Universidad Católica de Chilem. Santiago: Universidad Católica de Chile. Tesis para optar al grado de licenciado en enfermería.

González, S. (1998) «Modernidad, Ciudadanias y Etica: Relaciones Lógicas». En Revista OIKOS. N6. 43-51. Santiago.

Gysling . (1995) «La investigación social en salud reproductiva en Chile: panorama al inicio de los noventa». Santiago. FLACSO y Programa Interdisciplinario de Estudios de Género, Universidad de Chile.

Molina N. (1998) «Tipos de familia en Chile». Jornada Nacional de Salud Sexual y Reproductiva. ICMER.

OMS. (1988) «Salud Reproductivay situación de las Mujeres».

ONU (1995) «Resúmenes del Programa de acción de la Conferencia Internacional sobre Población y desarrollon. Naciones Unidas.

OPS (1992a) Documento de referencia «Salud y Autocuidado: Una toma de decisiones para la mujer frente a su vida, reflexiones para un debate».

OPS (1992b) Informe de Relatoria del Grupo de Trabajo sobre el tema de mujer, Salud y Autocuidado.

Sepúlveda R. (1991) «Autocuidado en salud: dinámica correctora de una medicina embriagada por la técnicam. EPAS, Vol VIII, $\mathrm{N} \times 3,36-37$.

SERNAM (1994) "Primer congreso de mujer y salud mental». Documento No35. Santiago.

Solimano, G. (1994) «Sexualidad y reproducción: hacia la construcción de derechos". Santiago. En: Sexualidad y Reproducción. CORSAPS. pp.7-10.

Tapia C.; Iturra V.; VarelaH. (1996) «Práctica de autocuidado en los profesionales de Antofagasta". Horizonte de Enfermería. Vol $7, \mathrm{~N}^{\mathrm{o}} 1$, pp.4-12.

UNICEF (1996) «Ser Mujer en los Noventa. Naciones Unidas.

Valdés T. (1998) «Modelos culturales vigentes y emergentes». Jornada Nacional de salud sexual y reproductiva. ICMER. Santiago.

Vidal P. (1998) «Realidad de la salud y los derechos reproductivos en Chile». Jornada Nacional de salud sexual y reproductiva. ICMER. Santiago. 